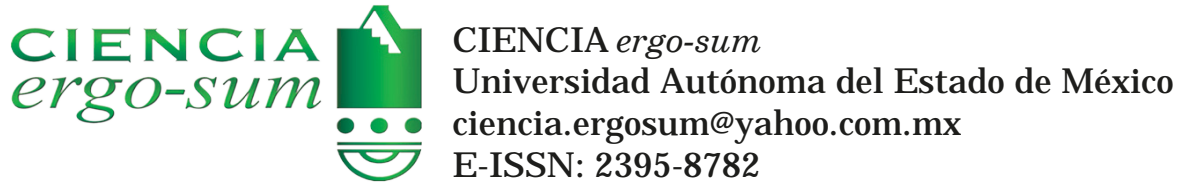

\title{
La confianza como determinante del compromiso, la in- tención de regreso y la recomendación del turista por un destino: el caso de Cancún
}

\author{
Matos Cámara, Rafael Fabricio
}

La confianza como determinante del compromiso, la intención de regreso y la recomendación del turista por un destino: el caso de Cancún

CIENCIA ergo-sum, vol. 26, núm. 3, noviembre 2019-febrero 2020|e58

Universidad Autónoma del Estado de México, México

Esta obra está bajo una Licencia Creative Commons Atribución-NoComercial-SinDerivar 4.0 Internacional.

Matos Cámara, R. F. (2019). La confianza como determinante del compromiso, la intención de regreso y la recomendación del turista por un destino: el caso de Cancún. CIE NCIA ergo-sum, 26(3). https://doi.org/10.30878/ ces.v26n3a1 


\title{
La confianza como determinante del compromiso, la intención de regreso y la recomendación del turista por un destino: el caso de Cancún
}

The trust as a determinant of commitment, intention to revisit and the tourist's recommendation to a destination: the case of Cancun

Rafael Fabricio Matos Cámara

Universidad La Salle Cancún, México

Recepción: 30 de enero de 2018

fabriciomat@hotmail.com

Aprobación: 20 de junio de 2018

\section{RESUMEN}

Se da a conocer en qué medida la confianza influye en el compromiso afectivo y en cómo determina la intención de regreso y la recomendación que los turistas españoles hace de Cancún a otros. Para tal fin, este estudio cuantitativo ha utilizado la metodología de ecuaciones estructurales. Como puntos a destacar se encuentra primero que las hipótesis han sido corroboradas al observar la influencia que tiene el compromiso afectivo sobre la recomendación, la cual es la relación más fuerte del modelo planteado, en segundo punto se observa la influencia que tiene la confianza sobre el compromiso afectivo y en tercero se demuestra, pero en menor grado, la influencia del compromiso afectivo sobre la intención de regreso al destino visitado.

Palabras ClaVe: confianza; compromiso afectivo, intención de regreso, recomendación, marketing de relaciones, destino turístico.

\begin{abstract}
The objective of this research is to know to what extent the trust influences the affective commitment and how the affective commitment determines the intention to re-visit and the recommendation that Spanish tourists make of Cancun to others. For this purpose, this quantitative study has used the methodology of structural equations. Finally, all the hypotheses have been corroborated observing the influence of the affective commitment on the recommendation, this being the strongest relationship of the proposed model. In the second point, the influence of trust on affective commitment is observed and, thirdly, it is demonstrated, but to a lesser degree, the influence of the affective commitment on the intention to revisit the above mentioned destination.
\end{abstract}

KEYWORDS: trust, affective commitment, intention to revisit, recommendation, relationship marketing, tourist destination.

\section{INTRODUCCIÓN}

La actividad turística se torna pujante en el mundo, ya que contribuye a potenciar la calidad de vida de los ciudadanos y el desarrollo económico de una región, además de compartirse la herencia cultural y gastronómica de los pueblos tras el arribo de los visitantes (Matos Cámara y San Martín Gutiérrez, 2006). De tal modo, durante la experiencia de visita los turistas experimentan una serie de percepciones y sensaciones al estar en contacto con el entorno (Rodríguez del Bosque y San Martín, 2008). Por lo tanto, la seguridad que el turista sienta por un destino tras las experiencias positivas obtenidas durante la visita será determinante en el desarrollo de la confianza, el compromiso afectivo, la repetición y la recomendación que lleva a cabo del lugar (Cristopher et al., 1991; Blazquez-Resino et al., 2015; Kozak, 2001; Morgan y Hunt, 1994; Prayag et al., 2017; Stylidis et al., 2017). En este sentido, se manifiesta la importancia del estudio de la visita del turista desde la óptica de la confianza y como factor determinante de la repetición y la recomendación por un territorio.

Por lo anterior, un factor que interviene en el establecimiento de las relaciones a largo plazo con un destino es la confianza como formadora del compromiso, recomendación e intención de regreso del turista (Matos Cámara 
y San Martín Gutiérrez, 2015). Es así que nos hacemos las siguientes preguntas: ¿cuáles son los determinantes que inciden en el visitante al momento de establecer una relación con el destino?, ¿qué factores influyen en la intención de regreso y la recomendación que el turista hace de un destino?, ¿en qué medida la confianza del consumidor por un destino influye en el compromiso, la intención de regreso y la recomendación por un destino? y ¿cómo la confianza del turista influye en el compromiso que siente por el destino? Así pues, estas interrogantes nos darán los lineamientos para realizar las hipótesis que deriven del modelo de investigación propuesto de cara a su contraste. De tales señalamientos, y con el propósito de contribuir al estudio del marketing de relaciones aplicado al contexto de los destinos turísticos, el objetivo de este artículo consiste en determinar en qué medida la confianza influye en el compromiso y de qué manera éste influye en la intención de regreso y la recomendación que el turista hace a otros del destino.

Para cumplir con nuestro objetivo, este trabajo se ha estructurado del siguiente modo: en la primera parte se presenta una introducción y justificación del problema tratado, las preguntas y el objetivo de la investigación. En segundo lugar se lleva a cabo una revisión de la literatura y se establecen los argumentos que dan cabida a las hipótesis. En tercer punto se plantea la metodología utilizada, el análisis factorial exploratorio y confirmatorio realizado para determinar el modelo global propuesto. En una cuarta parte se detallan las conclusiones e implicaciones profesionales derivadas de esta investigación.

\section{Marco teórico}

\section{1. El enfoque de marketing de relaciones}

Los destinos turísticos actualmente invierten en actividades orientadas a lograr la repetición de visitas y a estimular la confianza de los turistas actuales y potenciales (Fyall et al., 2003; Kozak, 2001; Matos Cámara y San Martín Gutiérrez, 2015; Blazquez-Resino et al., 2015). De este modo, los turistas buscan maximizar sus expectativas de valor a partir de sus experiencias. Por lo tanto, para lograr la confianza de los visitantes tras la repetición de la visita, los destinos ofrecen un elevado valor añadido (Kozak y Rimmington, 2000; Kozak, 2001; Kozak y Beaman, 2006). Debido a estos cambios, los destinos están experimentando una transformación, pues han pasado de ofertar solamente bienes y servicios para satisfacer el simple consumo al desarrollo de una experiencia de consumo que llevará a materializar las relaciones a largo plazo como reducto de la confianza formada de la seguridad que el turista perciba del lugar (Morgan y Hunt, 1994; Gummesson, 2008; Matos Cámara y San Martín Gutiérrrez, 2015).

Por consiguiente, y con miras a conseguir una experiencia de consumo integral, el enfoque de marketing sostiene que las relaciones para un destino deben ser consideradas como el esfuerzo integrado de los agentes implicados en el destino para identificar, mantener y crear una red personalizada con turistas por medio del acopio continuo de información para así elaborar estrategias de crecimiento y valor añadido a través de las interacciones durante un largo periodo (Blazquez-Resino et al., 2015; Fyall et al., 2003; Vargo y Lusch, 2004). De tal manera, para este artículo la confianza generada por los agentes del lugar resulta vital para el desarrollo de las relaciones que establecen los destinos con los visitantes, pero con una perspectiva de largo plazo. Por esta razón, Vargo y Lusch (2004) consideran que el marketing de relaciones es una perspectiva de mantenimiento y servicio continuo a través de la interacción permanente con el producto turístico y los agentes implicados. De esta forma, estos autores conciben que los servicios con un grado elevado de seguridad y honestidad integran el factor principal para crear valor y confianza del destino.

Sin duda alguna, los destinos dependen de un sinnúmero de factores ligados al contexto de la vivencia, los cuales contribuyen al éxito o al fracaso del marketing que específicamente construyan de la relación. Por ello, la existencia del compromiso y la confianza en la relación son dos variables clave (Morgan y Hunt, 1994). En este sentido, el compromiso y la confianza como reducto de la seguridad que el turista perciba en el destino por parte de sus agentes implicados son cruciales en la relación, puesto que los motivan a crear con él relaciones fuertes y duraderas (Morgan y Hunt, 1994; Fyall et al., 2003; Matos Cámara y San Martín Gutiérrez, 2015). A raíz de lo 
dicho, teorizamos que la presencia de la confianza y el compromiso afectivo en la relación es central a la comercialización que se realiza del destino. Por ello, este trabajo se cobija a partir de los postulados de Morgan y Hunt (1994) y parte de la teoría compromiso-confianza. Al respecto, el compromiso afectivo y la confianza conducen directamente a los comportamientos cooperativos de todos los agentes que interactúan en un destino de cara a crear seguridad en el turista y que son conducentes al éxito del marketing en la relación.

\section{Planteamiento de las hipótesis de trabajo}

\section{1. La confianza como determinante del compromiso afectivo del turista con el destino}

Para comprender de qué manera la confianza en las relaciones que establece un turista con el destino determina al compromiso, nos podemos basar en el modelo de marketing relacional desarrollado por Morgan y Hunt (1994), que señala la confianza como variable clave mediadora para el desarrollo de las relaciones con los clientes a largo plazo y se convierte en un factor determinante para conseguir el compromiso afectivo en una relación. Por su parte, la confianza que deposita el turista en un destino es la que disminuye la inseguridad percibida y el miedo al oportunismo de los diferentes agentes implicados en el lugar (San Martín, 2003), y propicia el desarrollo de niveles crecientes de compromiso afectivo en la relación (Fyall et al., 2003). En este sentido, una relación en donde las partes confían mutuamente genera suficiente valor como para que estén dispuestas a comprometerse (Blazquez-Resino et al., 2015; Eitzinger y Wiedemann, 2008; Matos Cámara y San Martín Gutiérrez, 2015; Gummesson, 2008). Por tanto, y derivado de estos argumentos, se propone la siguiente hipótesis de trabajo.

\section{H1: La confianza del turista por un destino influye positivamente en el compromiso afectivo del turista con ese destino.}

\section{2. El compromiso afectivo como determinante de la intención de regreso y la recomendación del turista con el destino}

Es vital el papel que manifiesta el compromiso afectivo para la seguridad relacional mediante los vínculos emocionales, empatía y cercanía existente entre las partes, además de que presenta escasa propensión al abandono y una característica duradera en el tiempo (Gilliland y Bello, 2002; San Martín, 2003; Fullerton, 2003). Por lo tanto, desempeña un papel notable en la disposición emocional del individuo por mantener una relación que mejora la percepción de valor futuro (Dick y Basu, 1994) con un territorio experimentado y su intención de regreso. Así, a partir de estas ideas proponemos la siguiente hipótesis.

H2: El compromiso afectivo del turista por un destino influye positivamente en la intención de regreso del turista a ese destino.

Siguiendo con los efectos del compromiso afectivo y como determinante de la recomendación del turista en la relación con un destino, los clientes que manifiestan un mayor grado de compromiso afectivo favorecerán un comportamiento boca-oreja (Brown et al., 2005). Esto se ha comprobado en diversos trabajos científicos (Hennig-Thurau et al., 2002; Lacey et al., 2007). Por ello, proponemos que el turista con mayor grado de compromiso afectivo tendrá una mayor probabilidad de transmitir una recomendación positiva del destino turístico. En este tenor, postulamos la siguiente hipótesis de trabajo.

H3: El compromiso afectivo del turista por un destino influye positivamente en la recomendación que realiza por ese destino. 
De tales argumentos, se presenta un modelo de relaciones causales con sus respectivas hipótesis de trabajo (figura 1).

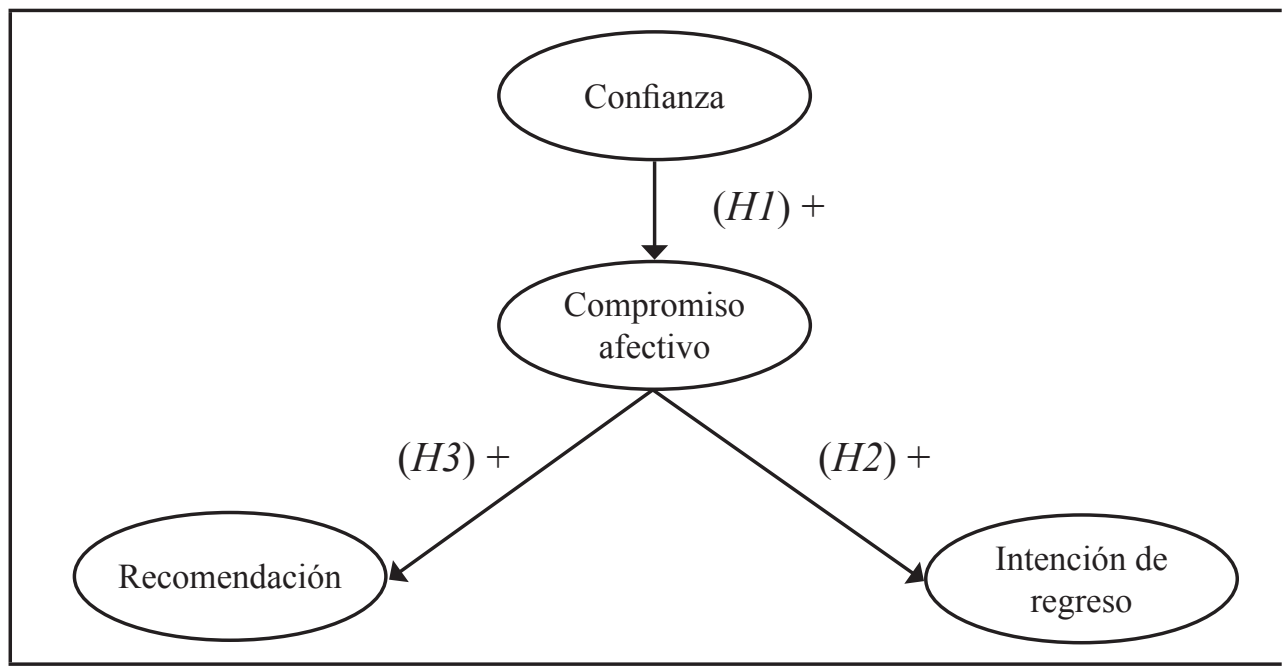

FIGURA 1

Modelo de relaciones causales propuesto

Fuente: elaboración propia.

\section{Metodología}

La investigación empírica se aplicará al estudio de las relaciones que establece el turista con un destino. Analizamos el caso de los turistas españoles que visitan Cancún.

\section{1. El universo de población y la muestra}

Para contrastar las hipótesis planteadas, se ha recogido información a través de encuestas personales que se aplicaron a los turistas españoles de manera aleatoria durante su visita. Hemos recolectado información a través de un cuestionario minuciosamente estructurado para ser aplicado a los visitantes. Para la selección de la muestra, hemos usado el método de poblaciones finitas (e.g. 111024 turistas españoles de vista en Cancún (tamaño de la población) (SIOM, 2016), que resulta en una muestra de 383 encuestas válidas de un total de 383 (personas de 18 a 60 años) (tasa de respuesta $=100 \%)$, las cuales han sido recopiladas en el aeropuerto de la ciudad de Cancún durante el trabajo de campo. Este método integra individuos más representativos y de diferentes características para estudiar el fenómeno de las visitas a un destino y su intención de repetir (Nusair et al., 2012). El error muestral es de 5.0\% (para el caso más desfavorable y un nivel de confianza de 95\%: $p=q=0.5$ ). A su vez, el periodo de realización de las encuestas se llevó a cabo entre septiembre y octubre de 2017.

\section{2. Medición de variables}

Para diseñar las escalas que nos permitieron elaborar el instrumento de recolección de datos (encuesta) recurrimos a los siguientes trabajos que proporciona la literatura. Para construir la escala de confianza se utilizaron los trabajos de Bart et al. (2005), Hewett y Bearden (2001), Roy et al. (2001) y San Martín (2003). Para el compromiso afectivo se consideraron los trabajos de Matos Cámara y San Martín Gutiérrez (2015), Garbarino y Johnson (1999) y Sirohi et al. (1998). Por su parte, la recomendación se ha hecho a partir de las aportaciones de Okazaki et al. (2007) y para la intención de regreso nos hemos basado en el trabajo de 
Bigné et al. (2001) y Leingpibul et al. (2009). En la tabla 1 se muestra la codificación de las variables e ítemes de la encuesta.

Respecto a las características personales del encuestado (sexo, estructura del hogar, edad, nivel de estudios, profesión y renta mensual neta del hogar), las escalas utilizadas son de elaboración propia y han sido adaptadas al entorno de estudio de un destino. Los ítemes que utilizaremos para medir las variables tratadas serán valorados a través de una escala tipo Likert de cinco posiciones (desde totalmente en desacuerdo hasta totalmente de acuerdo con la proposición formulada), excepto las de las variables sexo, estructura del hogar, edad, nivel de estudios, profesión y renta mensual neta del hogar.

La muestra está conformada por $58.7 \%$ hombres y $41.3 \%$ mujeres. En cuanto a la edad, $3.4 \%$ de los encuestados tiene menos de 20 años; $34.3 \%$ entre 21 y 30 años, que es el dato el dato de mayor frecuencia; $33 \%$ entre 31 y 40 años, el segundo más representativo; $17 \%$ entre 41 y 50 años; $7.0 \%$ entre 51 y 60 años y $5.4 \%$ más de 60 años. En estructura del hogar, 36.3\% vive de modo unipersonal; 31.4\% resulta ser pareja sin hijos; $26.8 \%$ lo constituyen las parejas con dos hijos y $5.5 \%$ más de tres hijos. Para nivel de estudios, $1.5 \%$ no cuenta con estudios; $5.9 \%$ tiene nada más el grado de secundaria terminada (FP); $7.5 \%$ cuenta con bachillerato; $17.5 \%$ tiene ciclos formativos; $31 \%$ ha resultado con el grado de universitarios medios y 37\% cuenta con el grado de universitarios superiores. De tal situación, destaca el grado de estudios universitarios en la muestra. En el caso de la profesión, 36.9\% ha resultado ser trabajador por cuenta ajena y representa el dato más notable de la muestra; $8.8 \%$ son empresarios; $10.8 \%$ los trabajadores autónomos; $6.7 \%$ jubilados; $17.8 \%$ funcionarios; $12.6 \%$ estudiantes; $1.8 \%$ amas de casa y $5 \%$ desempleados. En cuanto al ingreso mensual del hogar, $18 \%$ percibe menos de $900 € ; 15.3 \%$ entre $901-1$ $200 € ; 26 \%$ entre $1201-1500 €$, dato más representativo en este rubro; $16.2 \%$ entre $1501-2000 € ; 16 \%$ entre 2 001-3 $000 €$; 7\% entre $3001-4000 €$ y 2\% mayor de $4000 €$. En síntesis, de esta muestra de españoles los hombres en un 19\% son quienes viajan más al destino entre edades de 21 a 30 años y 31 a 40 años; en la estructura del hogar, las personas encuestadas viven de manera unipersonal, en un segundo grado han resultado ser parejas sin hijos y en tercer punto son parejas con hijos. A su vez, para el grado de estudios que poseen los turistas, en mayor grado cuentan con estudios universitarios superiores, y en profesión un dato robusto nos explica que la muestra está compuesta por trabajadores por cuenta ajena, y segundo lugar por funcionarios y en un tercer grado por estudiantes. Por último, el nivel de ingreso mensual del hogar más destacado resulta entre 1201-1 $500 €$.

Por otra parte, se ha elaborado un análisis factorial confirmatorio para el conjunto de variables observadas obteniendo resultados favorables por el número de factores que han resultado tal y como se habían proyecto (tabla 2). Del mismo modo, con el análisis factorial confirmatorio hemos podido comprobar la validez convergente y discriminante de las escalas de medida y depurarlas si fuere necesario hasta simplificarlas, aunque no ha sido nuestro caso, y así llegar a las definitivas con la ayuda del programa estadístico LISREL 8.7. Estas características de la comprobación de validez convergente y discriminante de las escalas de medida se convierten en una de las características más destacadas del programa estadístico LISREL y del porqué en esta investigación nos hemos decantado por su uso. Conjuntamente, el programa estadístico LISREL "ofrece una mayor variedad de métodos de estimación y cuenta con una interfaz gráfica que permite crear el modelo (path diagram) automáticamente, una vez que se lleva acabo el análisis estadístico de los datos" (Escobedo et al., 2016: 18).

De acuerdo con lo anterior, con el programa estadístico LISREL 8.7 hemos recurrido a las contribuciones de Jöreskog y Sörbom (2001), Babin et al. (2008) y Hair et al. (2009) sobre la modelización mediante ecuaciones estructurales para estimar nuestro modelo global. En este sentido, hemos corroborado en la literatura científica que son muchos los trabajos que han aplicado el método de ecuaciones estructurales usando complejos modelos que evalúan una gran cantidad de relaciones desde diferentes teorías y enfoques dentro de la disciplina del marketing turístico y que ha permitido en su mayor contribución el programa estadístico LISREL como la validación de escalas y la estimación de modelos globales por medio del análisis factorial confirmatorio y la confirmación de las hipótesis de trabajo (Jalilvand et al., 2012; Jalilvand et al., 2013; Matos Cámara y San Martín Gutiérrez, 2015; Nusair et al., 2012; San Martín, 2003; Stylidis et al., 2017). 
TABLA 1

Codificación de las variables e ítemes de la encuesta

\begin{tabular}{|c|c|c|c|}
\hline Variable & Cod. & Descripción ítem & Trabajos \\
\hline \multirow{5}{*}{ Confianza } & V1 & 1. Este destino cumple sus promesas & $\begin{array}{l}\text { Adaptado de Hewett y } \\
\text { Bearden }(2001)\end{array}$ \\
\hline & $\mathrm{V} 2$ & $\begin{array}{l}\text { 2. Las características que tiene este destino son acordes con } \\
\text { mis gustos y preferencias }\end{array}$ & \multirow{2}{*}{ Adaptado de Bart et al. (2005) } \\
\hline & $\mathrm{V} 3$ & $\begin{array}{l}\text { 3. Este destino es seguro y puede confiarse en él y en sus } \\
\text { servicios }\end{array}$ & \\
\hline & V4 & $\begin{array}{l}\text { 4. Creo que al surgir problemas los gestores de este destino } \\
\text { tendrán un comportamiento honesto conmigo }\end{array}$ & \multirow{2}{*}{ Adaptado de Roy et al. (2001) } \\
\hline & V5 & $\begin{array}{l}\text { 5. Creo que los gestores de este destino toman en consi- } \\
\text { deración las repercusiones que sus acciones pueden tener } \\
\text { sobre sus turistas }\end{array}$ & \\
\hline \multirow{4}{*}{$\begin{array}{l}\text { Compromiso } \\
\text { afectivo }\end{array}$} & V6 & 1. Me considero leal a este destino & $\begin{array}{l}\text { Adaptado de Matos Cámara y } \\
\text { San Martín Gutiérrez (2015) }\end{array}$ \\
\hline & V7 & 2. Siento un cierto afecto por este destino & $\begin{array}{l}\text { Adaptado de Garbarino y John- } \\
\text { son (1999) }\end{array}$ \\
\hline & V8 & 3. Defiendo a este destino cuando alguien lo critica & $\begin{array}{l}\text { Adaptado de Matos Cámara y } \\
\text { San Martín Gutiérrez (2015) }\end{array}$ \\
\hline & V9 & $\begin{array}{l}\text { 4. Mantener la relación con este destino es importante para } \\
\text { mí, pues me siento beneficiado de ello }\end{array}$ & Adaptado de Sirohi et al. (1998) \\
\hline \multirow{6}{*}{ Recomendación } & V10 & 1. Comentaré cosas positivas de este destino & \multirow{6}{*}{$\begin{array}{l}\text { Adaptado de Okazaki et al. } \\
\text { (2007) }\end{array}$} \\
\hline & V11 & 2. Recomendaré el destino a mis familiares y amigos & \\
\hline & V12 & 3. Recomendaré el destino a otras personas & \\
\hline & V13 & 4. Recomendaré el destino altamente hacia el futuro & \\
\hline & V14 & $\begin{array}{l}\text { 5. Difundiré información del destino para que todos mis } \\
\text { amigos lo conozcan }\end{array}$ & \\
\hline & V15 & $\begin{array}{l}\text { 6. Comunicaré a mi(s) amigo(s) que pudieran estar interesados } \\
\text { en la información del destino }\end{array}$ & \\
\hline
\end{tabular}

V17 1. Intentaré regresar a este destino en el futuro

Adaptado de Bigné et al. (2001)

Intención de regreso
V18 2

2. La próxima vez que acuda a un destino, es muy probable que regrese a él

V19 3. No creo que vuelva a acudir a este destino turístico

V20 4. Tras mi experiencia actual, creo que visitaré de nuevo este destino en el futuro

V21 Sexo Hombre/Mujer

V22 Edad

Características personales del encuestado Datos demográficos y socioeconómicos

V24 Nivel de estudios

V25 Profesión

\section{Menor de 20 años} a 50 años / De 51 a 60 años / Más de 61 años

Adaptado de Leingpibul et al. (2009)

V23 Estructura del hogar Unipersonal / Pareja sin hijos / Pareja con hijos
Sin estudios / Secundaria / Bachillerato / Ciclos formativos / Universitarios medios / Universitarios superiores Trabajador por cuenta ajena / Empresario / Autónomo / Jubilado (a) / Funcionario (a) / Estudiante /Ama de casa / Desempleado (a)

V26 Renta mensual del hogar 
TABLA 2

Resultados del análisis factorial confirmatorio

\begin{tabular}{|c|c|c|c|c|}
\hline Variables & Ítems de medidas & $\begin{array}{l}\text { Alfa de } \\
\text { Cronbach }\end{array}$ & $\begin{array}{c}\text { Cargas } \\
\text { factoriales }\end{array}$ & $\mathrm{KMO}$ \\
\hline \multirow{5}{*}{ Confianza } & 1. Este destino cumple sus promesas & \multirow{5}{*}{0.855} & 0.794 & \multirow{5}{*}{0.822} \\
\hline & $\begin{array}{l}\text { 2. Las características que tiene este destino son acordes con mis } \\
\text { gustos y preferencias }\end{array}$ & & 0.740 & \\
\hline & \multirow{2}{*}{$\begin{array}{l}\text { 3. Este destino es seguro y puede confiarse en él y en sus servicios } \\
\text { 4. Creo que al surgir problemas los gestores de este destino tendrán } \\
\text { un comportamiento honesto conmigo }\end{array}$} & & 0.802 & \\
\hline & & & 0.811 & \\
\hline & $\begin{array}{l}\text { 5. Creo que los gestores de este destino toman en consideración las } \\
\text { repercusiones que sus acciones pueden tener sobre sus turistas }\end{array}$ & & 0.831 & \\
\hline \multirow{4}{*}{$\begin{array}{l}\text { Compromiso } \\
\text { afectivo }\end{array}$} & 1. Me considero leal a este destino & \multirow{4}{*}{0.896} & 0.893 & \multirow{4}{*}{0.835} \\
\hline & 2. Siento un cierto afecto por este destino & & 0.877 & \\
\hline & 3. Defiendo a este destino cuando alguien lo critica & & 0.852 & \\
\hline & $\begin{array}{l}\text { 4. Mantener la relación con este destino es importante para mí, pues } \\
\text { me siento beneficiado de ello }\end{array}$ & & 0.882 & \\
\hline \multirow{6}{*}{ Recomendación } & 1.Comentaré cosas positivas del destino & \multirow{6}{*}{0.920} & 0.864 & \multirow{6}{*}{0.902} \\
\hline & 2. Recomendaré el destino a mis familiares y amigos & & 0.884 & \\
\hline & 3. Probablemente recomendaré el destino a otras personas & & 0.896 & \\
\hline & \multirow{2}{*}{$\begin{array}{l}\text { 4. La posibilidad de que recomiende el destino en el futuro es muy alta } \\
\text { 5. Difundiría la información del destino porque es importante que } \\
\text { todos mis amigos lo conozcan }\end{array}$} & & 0.830 & \\
\hline & & & 0.838 & \\
\hline & $\begin{array}{l}\text { 6. Comunicaré a mi(s) amigo(s) que pudieran estar interesados en la } \\
\text { información del destino }\end{array}$ & & 0.773 & \\
\hline \multirow{4}{*}{$\begin{array}{l}\text { Intención de } \\
\text { regreso }\end{array}$} & 1. Intentaré regresar a este destino en el futuro & \multirow{4}{*}{0.776} & 0.886 & \multirow{4}{*}{0.703} \\
\hline & $\begin{array}{l}\text { 2. La próxima vez que acuda a un destino, es muy probable que } \\
\text { regrese a este destino }\end{array}$ & & 0.776 & \\
\hline & 3. No creo que vuelva a acudir a este destino turístico & & 0.591 & \\
\hline & $\begin{array}{l}\text { 4. Tras mi experiencia actual, creo que visitaré de nuevo este destino } \\
\text { en el futuro }\end{array}$ & & 0.893 & \\
\hline
\end{tabular}

Fuente: elaboración propia.

A su vez, este tipo de análisis factorial nos ha permitido determinar el grado de validez de cada escala, lo cual es un concepto más amplio que el de fiabilidad. Asimismo, hemos utilizado las siguientes medidas de bondad del ajuste: el valor de chi-cuadrado $(p=0.0$ ), la raíz media estandarizada (RMSEA), el índice de bondad de ajuste comparativo (CFI), el índice de ajuste normado (NFI), el índice de ajuste incremental (IFI) y el índice de bondad de ajuste (GFI). En este caso, estos índices son positivos si están en el umbral o sobrepasan el valor de 0.9 (Mackenzie, 2001). A su vez, es adecuado que la RMSEA presente un valor igual o menor a 0.08 (Hair et al., 2009). Con estas medidas, hemos seguido la recomendación de los autores en cuanto a tomar diferentes tipos de medidas del ajuste con el objetivo de evitar los inconvenientes de cada una de ellas (Mackenzie, 2001). Al mismo tiempo, hemos realizado dos pruebas para valorar si las medidas son adecuadas al momento del análisis factorial confirmatorio dicho. En este caso, se ha calculado el coeficiente Alfa de Cronbach para conocer el grado de fiabilidad de las escalas y han resultado aceptables. También hemos realizado la prueba de Kaiser-Meyer-Olkin (KMO) que tiene valores entre 0 y 1 . En esta prueba, cuanto más cerca de 1 más ajustados serán los datos; al contrario, valores menores a 0.5 no se consideran pertinentes para la validación. En este modelo estructural hemos considerado de forma explícita la naturaleza de cada variable tratada y así testar la contrastación de las hipótesis planteadas que nos ha llevado a extraer las conclusiones generales del estudio. En resumen, para la estimación del modelo global se ha llevado a cabo un análisis factorial confirmatorio tomando en consideración los factores de medida que han sido estimados. De tal situación, el resultado de la bondad del ajuste del modelo ha resultado aceptable para validar las relaciones estudiadas. 
Resultados del análisis factorial confirmatorio:

$X^{2}=(3) 73.348(p=0.0) ; \mathrm{RMSEA}=0.070 ; \mathrm{NFI}=0.900 ; \mathrm{CFI}=0.900 ; \mathrm{IFI}=0.901 ; \mathrm{GFI}=0.908$

\section{Resultados de la estimación del modelo final}

En este apartado se comentarán los resultados de la estimación del modelo final, así como la confirmación de cada una de nuestras hipótesis de trabajo y los resultados más notables que se desprenden del modelo (figura 2).

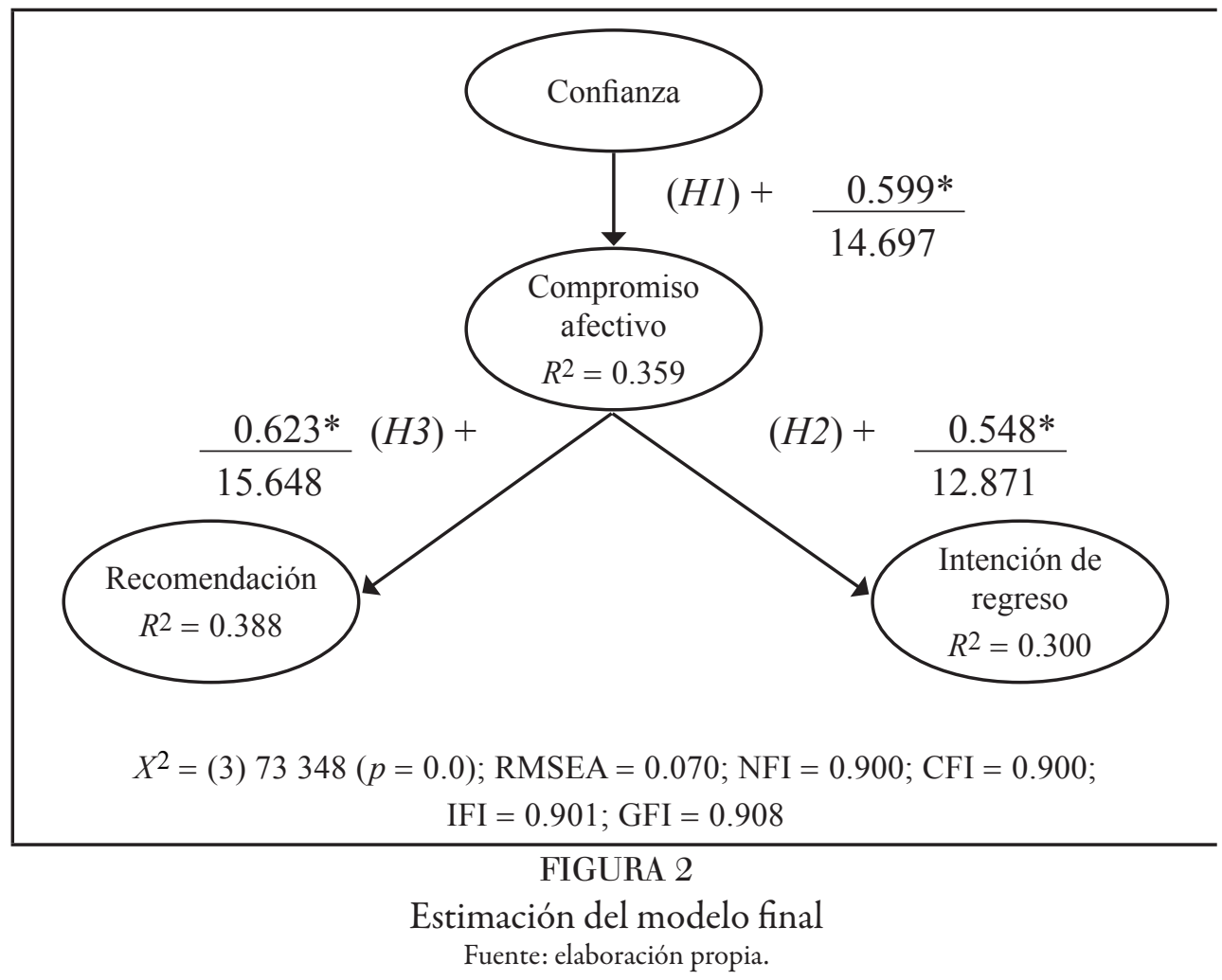

Nota: nivel de significancia ${ }^{*} p<0.05$; hipótesis confirmada.

Respecto a las hipótesis planteadas, todas ellas han sido aceptadas. La hipótesis $H 1$ se comprueba: la confianza que siente el turista por el destino construye el compromiso afectivo hacia este destino $(\lambda=0.599 ; t=14.697$; $p<0.05)$. Estos aportes comprueban la operatividad de la teoría compromiso-confianza propuesta por Morgan y Hunt (1994) y confirmada por otros autores como Garbarino y Johnson (1999); Gummesson (2008) y Roy et al. (2001) y llevada al estudio del marketing de destinos turísticos (Fyall et al., 2003; Matos Cámara y San Martín Gutiérrez, 2015). Esta hipótesis presenta el segundo valor más representativo para explicar las relaciones de marketing que establece el turista por el destino. Por otro lado, la hipótesis $H 2$ se confirma: el compromiso afectivo que siente el turista por el destino turístico visitado influye de manera directa y positiva en la intención de regreso $(\lambda=0.548 ; t=12.871 ; p<0.05)$, pero en menor medida que la recomendación que el turista hace a otros del destino visitado, es decir, que es la hipótesis que presenta el menor valor dentro del modelo propuesto para explicar las relaciones que establecen los visitantes españoles por el destino y de cara a determinar el grado en la intención de regresar. Finalmente, la hipótesis $H 3$ se corrobora: el compromiso afectivo que siente el turista por el destino influye de forma directa y positiva en la recomendación que lleva a cabo en sus familiares, amigos y conocidos del lugar visitado $(\lambda=0.623 ; t=15.648 ; p<0.05)$. 


\section{CONCLUSIONES E IMPLICACIONES PROFESIONALES, LIMITACIONES Y LÍNEAS FUTURAS DE INVESTIGACIÓN}

Para concluir, y como se ha podido constatar, la confianza que siente el turista español por Cancún como destino turístico influye de manera positiva en el compromiso afectivo que siente por él. Sin embargo, esta relación ocupa el segundo peldaño en el modelo investigado para crear un marketing de relaciones robusto, lo cual resulta implicante para los agentes (gestores) que interactúan en este destino. Por ello, el lugar estudiado tendrá que esforzarse aún más por desarrollar la confianza en sus turistas durante la visita. Por consiguiente, esto se logrará en un entorno en donde prevalezca la seguridad hacia los visitantes, las buenas prácticas en las transacciones turísticas, una infraestructura de la ciudad y conectividad de transporte adecuada, así como por evitar en todo momento los actos de corrupción de todos los agentes implicados (por ejemplo dependencias gubernamentales, hoteles, restaurantes, transportes, líneas aéreas, etc.) (Eitzinger y Wiedemann, 2008). Sin lugar a duda, todos estos factores alentarán la confianza por el destino turístico visitado tal y como se revela al comprobar la hipótesis $H 1$. Por su parte, se observa que el efecto de la recomendación que hace el turista del lugar a otros ha resultado tener la mayor fortaleza para explicar las relaciones por el destino visitado (e.g. Cancún). Así, el efecto boca-oreja resulta importante para establecer la recomendación por un destino al mostrar una fortaleza incalculable a diferencia de las campañas publicitarias mediáticas que se lleven a cabo del destino (Brown et al., 2005; Matos et al., 2014; Prayag et al., 2017). En añadidura, se comprueba que los clientes que manifiestan un mayor grado de compromiso afectivo favorecerán un comportamiento boca-oreja o recomendación positiva (Brown et al., 2005) tal y como se comprobó en la hipótesis H3. Por último, el compromiso afectivo, como se ha confirmado, influye en la intención de regreso (hipótesis H2). No obstante, pese a que la influencia es positiva, la intención de regreso ha resultado ser la relación con menos peso en el modelo. En cuanto a las implicaciones profesionales, los agentes del destino tendrán que desarrollar un clima donde prevalezca la seguridad y se fortalezca la confianza del turista por el lugar y la intención de regreso. A su vez, los gestores del destino deberán generar buenas vivencias a través del estímulo afectivo que el turista experimente con los diferentes productos turísticos que consuma durante su estancia, por lo que es importante trabajar en cooperación con todos los agentes implicados como lo indica el enfoque stakeholder (Friedman y Miles, 2002). En este sentido, los stakeholders (e.g. grupos de interés) se explican como cualquier agente o individuo que puede afectar o es afectado por el logro de los objetivos de la organización tanto de forma interna y externa (Freeman, 1984; Mitchell y Cohen, 2006; Sirgy, 2002; Yilmaz y Gunel, 2009). A su vez, el término agente implicado hace referencia a los diversos intereses que participan en un destino turístico como Cancún. Así, los grupos de agentes implicados de cualquier destino representan una amplia y diversa gama de intereses, dado que cada uno tiene su propio conjunto único de expectativas, necesidades y valores. Respecto al destino tratado, los gestores deberán realizar acciones cooperadas de forma interna con accionistas, agentes financieros, intermediarios, clientes internos, el propio gobierno, proveedores, empleados, medios de comunicación inmersos en él y competidores. Al mismo tiempo, y de manera externa, deberán maximizar sus acciones con asociaciones comerciales, universidades, grupos pilotos, grupos de activistas, clientes externos y grupos defensores de los clientes (Mitchell y Cohen, 2006; Sirgy, 2002; Yilmaz y Gunel, 2009). En resumen, lo que hace especial al sector turístico por encima de otros es su estructura muy fragmentada y diversa que requiere de acciones cooperadas para tener éxito. De tal situación, existen ciertos factores que dentro de sus implicaciones profesionales Cancún necesitará atender bajo el enfoque stakeholder: a) el sector turístico es un gran sistema que necesita estar coordinado por cada una de los agentes que lo integran, $b$ ) comprender la interdependencia del trabajo de los agentes implicados es crucial (empresas), c) los destinos son dinámicos y se encuentran en constante cambio, $d$ ) el sector turístico es complejo y consiste en diferentes tipos de actividades y organizaciones y e) el destino estudiado deberá evaluar día a día que la competencia en el turismo es intensa (Yilmaz y Gunel, 2009).

Por su parte, las limitaciones de este trabajo radican en que es un estudio de corte transversal, ya que únicamente se evalúan los efectos de las variables estudiadas en un periodo específico y se ciñe en un segmento de mercado como es el mercado español. En el tema de líneas futuras de investigación será interesante de valorar a 
otros mercados y a otro tipo de variables de trabajo como la publicidad, los precios y la satisfacción para conocer cómo influyen en la recomendación y la intención de regresar a Cancún como visitante.

\section{AnÁLISIS PROSPECTIVo}

En añadidura, dentro de un análisis prospectivo, la interrogante que nos viene a la mente es ¿cómo los resultados obtenidos en esta investigación pueden contribuir a implementar un marketing eficaz del destino ante la percepción del turista? Es así que los agentes implicados deberán generar oportunas estrategias de marketing de relaciones y trabajar bajo el enfoque stakeholder (mercados ampliados) y en cooperación de todos los agentes implicados por la misma naturaleza tan fragmentada que impera en el producto turístico (Morgan y Hunt, 1994; Friedman y Miles, 2002; Ford, 2011). Además, hemos comprobado que es necesario proveer de seguridad al turista durante su visita en el destino para que esto permita desarrollar experiencias únicas e irrepetibles y también estimular el compromiso afectivo o emocional del visitante. Todo lo anterior es crucial para despertar la recomendación y la intención de regreso del visitante (Blazquez-Resino et al., 2015; Ford, 2011; Matos Cámara y San Martín Gutiérrez, 2015; Stylidis et al., 2017). A su vez, el destino requiere de mayor innovación, especialización y tematización de los diferentes servicios e infraestructura turística. A pesar de que Cancún es un buen lugar para visitar, hay que considerar que al turista le agrada tener una experiencia integral, ya que gusta de viajar a otros lugares cercanos como Playa del Carmen y Tulum para experimentar diferentes vivencias. Por tanto, la oferta integrada de circuitos turísticos puede contribuir a generar mayores niveles de estancia y gasto y a estimular el desarrollo de la confianza mediante la buena gestión de todos los agentes implicados en el territorio ante la percepción del turista. Por último, las vivencias únicas experimentadas por los visitantes harán que ellos recomienden el destino a sus amigos, conocidos y familiares y traigan como resultado un efecto de alta notoriedad ante turistas potenciales más que los efectos de la publicidad tradicional y las costosas campañas publicitarias de corte transaccional y que poco involucran al turista para el establecimiento de sus relaciones con el destino a largo plazo (Matos et al., 2014).

\section{Agradecimientos}

A todos aquellos turistas que con su apoyo han contribuido a la realización de este trabajo de investigación y a los revisores de la revista CIENCIA ergo-sum.

\section{REFERENCIAS}

Babin, B. J., Hair, J. F. y Boles, J. S. (2008). Publishing research in marketing journals using structural equations modeling. Journal of Marketing Theory and Practice, 16(4), 279-285.

Bart, Y., Shankar, V., Sultan, F. y Urban, G. L. (2005). Are the drivers and role of online trust the same for all Web sites and consumers? A large-scale exploratory empirical study.Journal of Marketing, 69(4), 133-152.

Bigné, J. E., Sánchez, M. I. y Sánchez, J. (2001). Tourism image, evaluation variables and after-purchase behavior: inter-relationship. Tourism Management, 22(6), 607-616.

Blazquez-Resino, J. J., Molina, A. y Esteban-Talaya, A. (2015). Service-dominant logic in tourism: The way to loyalty. Current Issues In Tourism, 18(8), 706-724.

Brown, T. J., Barry, T. E., Dacin, P. A. y Gunst, R. F. (2005). Spreading the word: Investigating antecedents of consumers' positive word-of-mouth intentions and behaviors in a retailing context. Journal of the Academy of Marketing Science, 33(2), 123-138. 
Cristopher, M., Payne, A. y Ballantyne, D. (1991). Relationship marketing. UK: Oxford.

Dick, A. y Basu, K. (1994). Customer loyalty: Toward an integrated conceptual framework. Journal of the Academy of Marketing Science, 22(2), 99-113.

Eitzinger, C. y Wiedemann, P. M. (2008). Trust in the safety of tourist destinations: hard to gain, easy to lose? New insights on the asymmetry principle. Risk Analysis: An International Journal, 28(4), 843-853.

Escobedo, M. T., Hernández, J. A., Estebané, V. y Martínez, G. (2016). Modelos de ecuaciones estructurales: características, fases, construcción, aplicación y resultados. Ciencia y Trabajo, 18(55), 16-22.

Ford, R. C. (2011). Friends to grow and foes to know: Lessons in successful stakeholder management from Orlando. International Journal of Contemporary Hospitality Management, 23(5), 696-712.

Freeman, R. E. (1984). Strategic Management: A Stakeholder Approach. Boston: Pitman.

Friedman, A. y Miles, S. (2002). Developing stakeholder theory. Journal of Management Studies, 39(1), 1-21.

Fullerton, G. (2003). When does commitment lead to loyalty? Journal of Service Research, 5(4), 333-344.

Fyall, A., Callod, C. y Edwards, B. (2003). Relationship marketing: The challenge for destinations. Annals of Tourism Research, 30(3), 644-659.

Garbarino, E. y Johnson, M. S. (1999). The different roles of satisfaction, trust and commitment in customer relationship. Journal of Marketing, 63(2), 70-87.

Gilliland, D. I. y Bello, D. C. (2002). Two sides to attitudinal commitment: the effect of calculative and loyalty commitment on enforcement mechanisms in distribution channels. Journal of the Academy of Marketing Science, 30(1), 24-43.

Gummesson, E. (2008). Total relationship marketing. Oxford: Butterworth-Heinemann.

Hair, J. F., Anderson, R. E., Tatham, R. L. y Black, W. C. (2009). Multivariate data analysis: A global perspective (7th ed). Upper Saddle River: Prentice Hall.

Hennig-Thurau, T., Gwinner, K. P. y Gremler, D. D. (2002). Understanding relationship marketing outcomes: An integration of relational benefits and relationship quality. Journal of Service Research, 4(3), 230-247.

Hewett, K. y Bearden, W. O. (2001). Dependence, trust, and relational behavior on the part of foreign subsidiary marketing operations: Implications for managing global marketing operations. Journal of Marketing, 65(4), 51-66.

Jalilvand, M. R., Ebrahimi, A. y Samiei, N. (2013). Electronic word of mouth effects on tourists' attitudes toward Islamic destinations and travel intention: An empirical study in Iran. Procedia-Social and Behavioral Sciences, 81, 484-489.

Jalilvand, M. R., Samiei, N., Dini, B. y Manzari, P. Y. (2012). Examining the structural relationships of electronic word of mouth, destination image, tourist attitude toward destination and travel intention: An integrated approach. Journal of Destination Marketing \& Management, 1(1-2), 134-143.

Jöreskog, K. G. y Sörbom, D. (2001). LISREL. Version 8.51. Chicago: Scientific Software International.

Kozak, M. (2001). Repeaters' behaviour at two distinct destinations. Annals of Tourism Research, 28(3), 784-807.

Kozak, M. y Beaman, J. (2006). Relationship between customer satisfaction and loyalty. Tourism Analysis, 11(6), 397-409.

Kozak, M. y Rimmington, M. (2000). Tourist satisfaction with Mallorca, Spain, as an off-season holiday destination. Journal of Travel Research, 38(3), 260-269.

Lacey, R., Suh, J. y Morgan, R. M. (2007). Differential effects of preferential treatment levels on relational outcomes. Journal of Service Research, 9(3), 241-256. 
Leingpibul, T., Thomas, S., Broyles, S. A.y Ross, R.H. (2009). Loyalty's influence on the consumer satisfaction and (re) purchase behavior relationship. Journal of Consumer Satisfaction, Disatisfaction and Complaining Behavior, 22(36).

Mackenzie, S. B. (2001). Opportunities for improving consumer research through latent variable structural equation modeling. Journal of Consumer Research, 28(1), 159-166.

Matos Cámara, R. F. y San Martín Gutiérrez, S. (2006). La confianza, la intención de compra, la reputación y las emociones en la relación del consumidor con la marca: aplicación al caso de la marca "Mundo Maya-México". Documentos de Trabajo Universidad de Burgos.

Matos Cámara, R. F. y San Martín Gutiérrez, S. (2015). Un modelo multidisciplinar para el estudio de la relación del consumidor español con un destino. Aplicación al caso de Mundo Maya México. DOCFRADIS Working Papers, 1503. Cátedra Fundación Ramón Areces de Distribución Comercial.

Matos, R. F., Jiménez, A., Ocampo, M. V. y Recio, M. (2014). La señalización como determinante de la satisfacción del turista español en un territorio: el caso de Quintana Roo. CIENCIA ergo-sum, 21(3), 217-229.

Mitchell, R. K. y Cohen, B. (2006). Stakeholder theory and the entrepreneurial firm. Journal of small Business Strategy, 17(1), 1-16.

Morgan, R. M. y Hunt S. D. (1994). The commitment-trust theory of relationship marketing. Journal of Marketing, 58(3), 20-38.

Nusair, K. K., Bilgihan, A., Okumus, F., \& Cobanoglu, C. (2012). Generation Y travelers' commitment to online social network websites. Tourism Management, 30, 1-10.

Okazaki, S., Katsukura, A., \& Nishiyama, M. (2007). How mobile advertising works: The role of trust in improving attitudes and recall. Journal of Advertising Research, 47(2), 165-178.

Prayag, G., Hosany, S., Muskat, B., \& Del Chiappa, G. (2017). Understanding the relationsaships between tourists' emotional experiences, perceived overall image, satisfaction, and intention to recommend. Journal of Travel Research, 56(1), 41-54.

Rodríguez del Bosque, I. R. y San Martín, H. (2008). Tourist satisfaction a cognitive-affective model. Annals of Tourism Research, 35(2), 551-573.

Roy, M., Dewit, O. y Aubert, B. (2001). The impact of interface usability on trust in web retailers. Internet Research: Electronic Networking Applications and Policy, 11(5), 388-398.

San Martín, S. (2003). La relación del consumidor con las agencias de viajes. Burgos: Servicios de publicaciones, Universidad de Burgos.

Sirgy, M. J. (2002). Measuring corporate performance by building on the stakeholders model of business ethics. Journal of Business Ethics, 35(3), 143-162.

Sirohi, N., Mclaughlin, E. W. y Wittink, D. R. (1998). A model of consumer perceptions and store loyalty intentions for a supermarket retailer. Journal of Retailing, 74(2), 223-245.

SIOM (Sistema Integral de Operación Migratoria). (2016). Número de visitantes internacionales que arribó a México vía aérea. México: Boletín del Instituto Nacional de Migración.

Stylidis, D., Shani, A. y Belhassen, Y. (2017). Testing an integrated destination image model across residents and tourists. Tourism Management, 58, 184-195.

Vargo, S. L., \& Lusch. R. F. (2004). Evolving to a new dominant logic for marketing. Journal of Marketing, 68(1), 1-17.

Yilmaz, B. S., y Gunel, O. D. (2009). The importance of strategic stakeholder management in tourism sector: Research on probable applications. Tourismos: An International Multidisciplinary Journal of Tourism, 4(1), 97-108. 